\title{
Vertebral compression fractures in multiple myeloma: redefining the priorities during the COVID-19 pandemic
}

\author{
P. D. Giorgi ${ }^{1}$ - G. R. Schirò ${ }^{1}$ - D. Capitani ${ }^{1}$ - G. D'Aliberti ${ }^{2}$ - E. Gallazzi ${ }^{1}$ (i)
}

Received: 23 April 2020 / Accepted: 2 May 2020 / Published online: 14 May 2020

(c) Springer Nature Switzerland AG 2020

Keywords Novel Coronavirus 2019 · SARS-CoV2 · Multiple myeloma $\cdot$ Kyphoplasty · Spine

\section{Introduction}

We are currently living an unprecedented situation for the modern medical profession. The Novel Coronavirus 2019 (COVID-19) has spread all over the world and has reached the epidemiologic criteria to be defined as a pandemic. In this dreadful situation, standard medical practice was markedly affected, giving priority to the treatment of COVID-19 patients, while maintaining regular care only for urgent nonCOVID conditions. However, a clear consensus on what is to be considered urgent is missing, with several expert-opinion and proposal published but often conflicting [1,2].

Multiple myeloma (MM) is a neoplastic disease of B-cell origin. Its main histological feature is the accumulation of malignant plasma cells in the bone marrow. Its reported incidence ranges between 2 and 3 per 100,000 in the general population. Bone involvement at presentation can be found in 70 to $100 \%$ of patients: indeed, it is included in the CRAB (hypercalcemia, renal failure, anemia, bony lesions) acronym of the most frequent presenting symptoms. Lytic bone disease is caused by plasma cells infiltration of the bone marrow and increased bone resorption; the osteolysis in turn causes severe bone pain, pathological fractures and, when occurring in the spine, also possible neurological compromise. Spine is the most common site of bony localization, with vertebral osteolytic lesions found in $70 \%$ of cases.

E. Gallazzi

enrico.gallazzi@gmail.com

1 Orthopedics and Traumatology Unit, Emergency and Urgency Department, A.S.S.T. Grande Ospedale Metropolitano Niguarda, Piazza dell'Ospedale Maggiore 3, Milan, Italy

2 Neurosurgery Unit, Emergency and Urgency Department, A.S.S.T. Grande Ospedale Metropolitano Niguarda, Milan, Italy
Indeed, bone involvement is the main cause of morbidity and mortality in MM patients [3].

Here we present a case of symptomatic vertebral compression fractures (VCFs) in an elderly patient positive for COVID-19 with newly diagnosed MM, treated with kyphoplasty. Our aim is to highlight the benefit of early treatment of this condition, which is being considered non-urgent during the current pandemic.

\section{Case presentation}

A spine surgery consultation was requested by the hematologist for a 79-year-old female with a newly diagnosed multiple myeloma (MM). The chief complaint was dorsolumbar pain of abrupt onset. The patient had a previous positive swab test result for COVID-19: indeed, since the pandemic outbreak, in our Institution each patient requiring Oncohematologic treatment is routinely screened to be addressed to either COVID-19 positive or negative specific pathway.

Her medical history consisted of a previous diagnosis of breast cancer, treated with quadrantectomy and local radiotherapy 6 years before. During the latest follow up visit, no sign of disease recurrences was found. She reported no previous back surgeries.

Diagnosis of multiple myeloma was established on the basis of the presence of a monoclonal peak at serum electrophoresis and a confirmative bone marrow biopsy.

The pain was progressive and not responding to conservative medical management (analgesics and bracing), with a Visual Analogue Scale (VAS) at rest of 8/10. Physical examination revealed only tenderness at lumbar spinous process with no signs of neurological deficit. Thoracolumbar X-rays showed VCF of T12, L2 and L4. A full spine MRI was obtained given the high sensitivity in identifying bony involvement in MM. Contrast-enhanced MRI was deemed 
unnecessary given that the diagnosis of MM was already established. The MRI showed a typical, variegated or "salt and pepper" pattern with diffuse small bone marrow focal lesions. Moreover, fat suppression sequences (STIR) showed marked hyperintensity compatible with recent fractures in T12, L1 and L2; on the contrary, no hyperintensity was observed in L4, thus suggesting an old osteoporotic fracture. No other fractures were found. On the basis of this, a diagnosis of pathological fractures in MM was made.

At the time of evaluation, the respiratory symptoms were mild, with no requirement of oxygen supplementation and an ambient air saturation of $98 \%$. A palliative percutaneous balloon kyphoplasty was proposed during a multidisciplinary discussion with the hematologist, the infectious disease specialist and the anesthesiologist.

According to our new COVID-19 reorganization, the patient followed the 'dirty' pathway. For the whole surgical procedure, the number of people in the OR was reduced at the necessary minimum; Anesthesiologist, nurses and surgeon were dressed properly with protective equipment: triple gloves, FFP2 surgical face masks and goggles or face shield. In OR, the patient was positioned prone on a radiolucent table. The procedure was performed with local anesthesia and under general sedation. An augmentation procedure with transpedicular biportal kyphoplasty of T12, L1 and L2 was performed using dedicated disposable tools. A total of about $7.5 \mathrm{ml}$ polymethylmethacrylate (PMMA) bone cement was used. At the end of the procedure, a final fluoroscopic and AP and LL X-rays control were obtained (Fig. 1).

Weight bearing without brace was allowed three hours after the procedure. The patient-reported significant pain reduction and was discharged after the first postoperative day, reporting a VAS of 3/10. Three days later, in accordance with the Hematologist, the patient resumed outpatient chemotherapy.

As of today, no worsening of respiratory SARS-CoV2 symptoms was recorded, and the patient resumed her daily life activities without brace and analgesics.
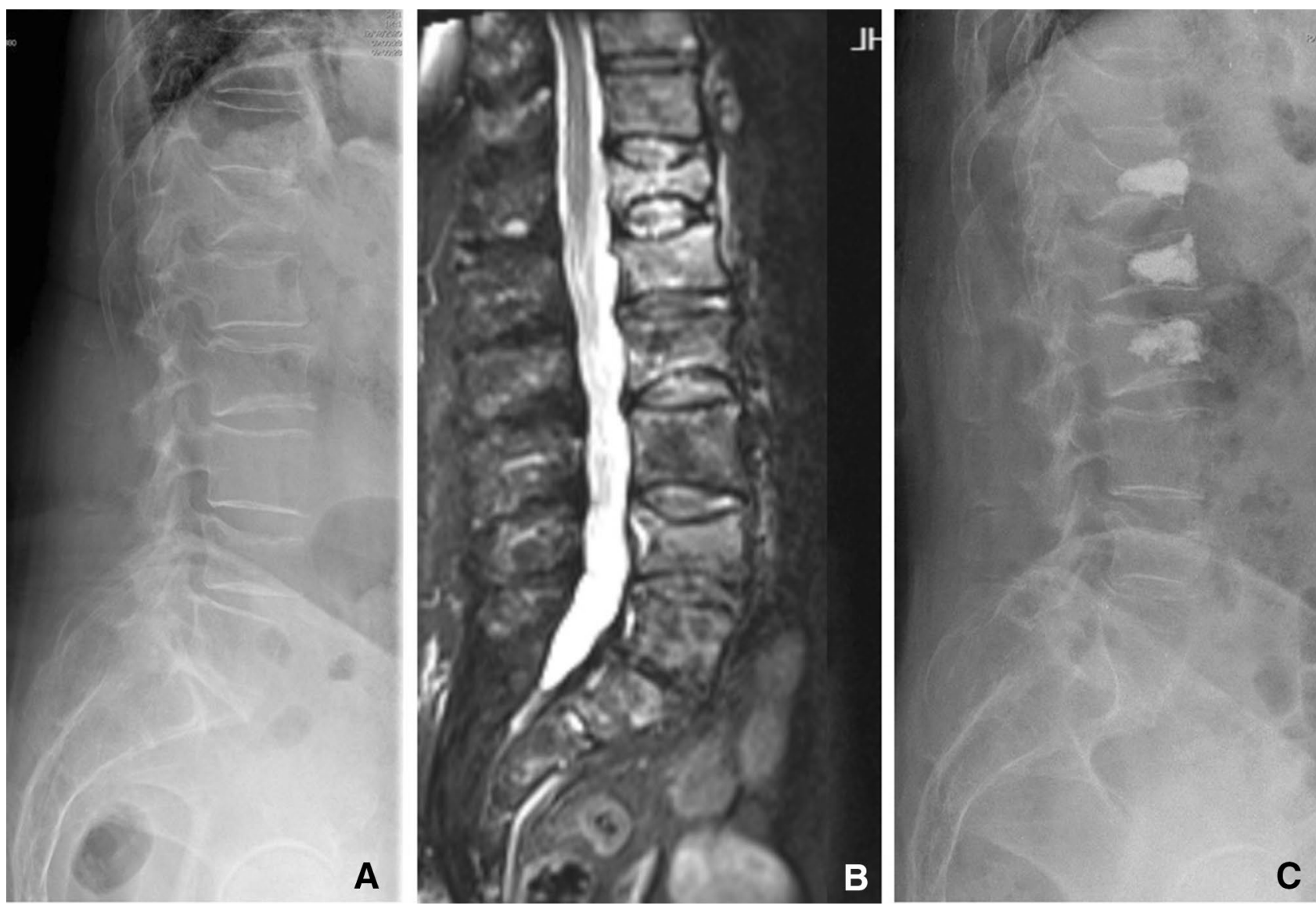

Fig. 1 Panel A, preoperative lateral X-ray showing fracture of T12, L1 and L2; Panel B, preoperative sagittal STIR MRI showing typical, variegated or "salt and pepper" pattern with innumerable small bone marrow focal lesions and hyperintensity in T12, L1 and L2; Panel C, postoperative lateral X-rays showing Kyphoplasty in T12, L1 and L2 


\section{Discussion}

The goal of the treatment of VCFs in MM is to keep the patient pain free and ambulatory, which should improve the adhesion to the frequent outpatient visits and chemotherapy treatment rounds. Conservative treatment with brace and analgesics has several limitations, such as local and systemic side effects, risk of deformity progression and pseudoarthrosis that may result in chronic pain. In this context, Percutaneous augmentation with vertebroplasty or kyphoplasty was shown to be a safe and effective, giving excellent pain relief and return to a near-normal level of function within a very short time. This treatment modality is supported by the Cancer Patient Fracture Evaluation (CAFE) prospective randomized controlled trial, which analyzed clinical outcomes of 134 patients with pathological VCFs [4]. In this trial, 49 of the patients had VCF in $\mathrm{MM}$, and were randomized either to the kyphoplasty group or to the non-surgical management group. The results of this trial showed more rapid and sustained pain relief, and thus reduced use of analgesics and bed rest days, after kyphoplasty.

The International Myeloma Working Group recently released a consensus statement on the role of balloon kyphoplasty in VCF in elderly patients. They consider persistent significant pain from an acute $\mathrm{VCF}$, or pain that limits activity or results in disability in the setting of a new event, as absolute indications. They also highlighted the importance of timing of balloon kyphoplasty, underlining that pain reduction can be extremely beneficial for a patient who will undergo a long and potentially heavy medical treatment such as chemotherapy.

In the case presented here, we performed a kyphoplasty to the vertebral levels with recent fractures, which were considered as the source of the acute pain. The choice of performing a kyphoplasty instead of vertebroplasty was based on the reports indicating a reduced risk of cement leakage and an increased capability of deformity correction for kyphoplasty.

\section{Rationale for the treatment during the COVID-19 pandemic}

Orthopedic practice has radically changed during the COVID-19 pandemic, mainly because National Health Systems underwent several reorganizational changes to adapt to the new necessities, mainly focusing on increasing the available intensive care unit beds and effectively allocating human and structural resources for treating COVID-19 patients. In Italy, to reach those goals, elective surgery has been deferred, and only trauma surgery has been allowed to continue. Recently, several papers have been published from groups all over the world, proposing different algorithms to establish which orthopedics procedures cannot be postponed. We have previously reported our early experience of emergency spinal surgery during the first month of this pandemic; where we presented the diagnostic and management algorithm used to manage each spinal emergency in our Institution. This algorithm allowed us to significantly reduce surgical waiting times while maintaining the surgeon and patient safety; nevertheless, the case mix described in our previous paper, consisted in traumatic vertebral fractures or cord compression due to spondylodiscitis or neoplasms [5]. Indeed, other reports on urgent spine surgery during the pandemic highlighted the benefit of treating two broad categories of spinal disease as urgencies: first, acute traumatic fractures with spinal instability; and second, spinal cord or nerve root compression with severe pain or neurological deficit $[1,2]$. In contrast, the case presented in this report cannot be included in the aforementioned categories; moreover, kyphoplasty in MM is usually postponed and undertaken only after failure of conservative treatment. Nonetheless, our decision making was based on several considerations: first, conservative treatment with bed rest immobilization and bracing can hamper the respiratory mechanics; furthermore, it limits the functional capabilities needed to undertake the frequent outpatient controls. Second, percutaneous kyphoplasty gives quick pain relief and fracture mechanical stability allowing an early and free mobilization of the patient and avoiding bed rest related complications. Finally, the patient could be quickly discharged after the surgery, therefore not overloading health-care resources and with a limited risk of nosocomial spreading of COVID-19.

\section{Conclusions}

Kyphoplasty is considered a non-urgent surgical treatment. Thus, these procedures have been deferred as a response aimed to optimize resources in the context of the COVID pandemic. Answering our initial question on what is deferable during COVID-19 pandemic, we wanted to highlight how a non-considered urgent procedure such as kyphoplasty can be the key for a right treatment. Indeed, in this case report, our decision making was not in accordance with an expert opinion on what is considered as urgent spinal surgery but was instead tailored to the specific needs of the patient. In these trying times, the physician should remember that sometimes urgency is not an absolute, but rather a relative concept. 
Acknowledgements The authors wish to thank Guillermo Sanchez Rosenberg MD for his critical review of the manuscript.

\section{Compliance with ethical standards}

Conflict of interest The authors declare that they have no conflict of interest.

Statement of human and animal rights All procedures followed were in accordance with the ethical standards of the Helsinki Declaration of 1975 , as revised in 2000 .

Informed consent Informed consent was obtained from the patient for being included in the study.

\section{References}

1. DePhillipo NN, Larson CM, O'Neill OR et al (2020) Guidelines for ambulatory surgery centers for the care of surgically necessary/ time-sensitive orthopaedic cases during the COVID-19 pandemic. J Bone Jt Surg Am. https://doi.org/10.2106/JBJS.20.00489

2. Ghogawala Z, Kurpad S, Falavigna A et al (2020) Editorial. COVID-19 and spinal surgery. J Neurosurg Spine. https://doi. org/10.3171/2020.4.SPINE20468

3. Panaroni C, Yee AJ, Raje NS (2017) Myeloma and bone disease. Curr Osteoporos Rep 15:483-498

4. Berenson J, Pflugmacher R, Jarzem P et al (2011) Balloon kyphoplasty versus non-surgical fracture management for treatment of painful vertebral body compression fractures in patients with cancer: a multicentre, randomised controlled trial. Lancet Oncol 12:225-235

5. Giorgi PD, Villa F, Gallazzi E et al (2020) The management of emergency spinal surgery during the COVID-19 pandemic in Italy. Bone Jt J. https://doi.org/10.1302/0301-620X.102B6 .BJJ-2020-0537

Publisher's Note Springer Nature remains neutral with regard to jurisdictional claims in published maps and institutional affiliations. 